\title{
Changing the Probability versus Changing the Reward
}

\author{
David M. Bruner ${ }^{\dagger}$
}

This version: January 2009

\begin{abstract}
There are two means of changing the expected value of a risk: changing the probability of a reward or changing the reward. Theoretically, the former produces a greater change in expected utility for risk averse agents. This paper uses two formats of a risk preference elicitation mechanism under two decision frames to test this hypothesis. After controlling for decision error, probability weighting, and order effects, subjects, on average, are slightly risk averse and prefer an increase in the expected value of a risk due to increasing the probability over a compensated increase in the reward. There is substantial across-format inconsistency but very little within-format inconsistency at the individual level.
\end{abstract}

Keywords: risk, uncertainty, experiments

JEL classification: C91, D81

\footnotetext{
${ }^{\dagger}$ Department of Economics, Appalachian State University, 3111 Raley Hall, 416 Howard St., Boone, NC 28608. E-mail address: brunerdm@appstate.edu. This research was undertaken at the University of Calgary Behavioural and Experimental Economics Laboratory (CBEEL). I would like to thank Christopher Auld, John Boyce, Glenn Harrison, Michael McKee, Bill Neilson, Rob Oxoby, Christian Vossler, Nat Wilcox, and two anonymous referees for their many helpful comments and suggestions. I would also like to thank participants at the 2007 North American Economic Science Association Meetings where an earlier version of this paper was presented.
} 


\section{Introduction}

Any gamble is composed of a set of possible outcomes and a probability distribution over those outcomes. ${ }^{1}$ The expected value of a gamble may be changed equivalently by changing the set of outcomes, the probability distribution, or both. Equivalent changes in the expected value, however, do not imply equivalent changes in risk. Suppose the expected value of a gamble is increased by changing the probability distribution and, equivalently, by changing the set of outcomes. The former produces a greater increase in expected utility (EU) than the latter for a risk averse agent. ${ }^{2}$ The question is, do risk averse people actually prefer changing the probability to changing the reward? EU theory says they will. Nonetheless, there is little definitive evidence to support this prediction since naturally occurring data do not satisfy the strict requirements necessary for an empirical investigation. A commonly used laboratory method for eliciting risk preferences can, however, allowing for a direct test of this implication of EU theory.

The most commonly used method of eliciting risk preference requires a respondent to make a series of dichotomous choices over lottery pairs. As the respondent proceeds through the series, the lotteries' expected values are increased to eventually induce her to switch from choosing the less risky to the more risky choice. The point at which she switches provides an estimate of risk preference. An attractive feature of this mechanism is the ability to control how the expected values are increased through the series. The researcher can manipulate either the probability distribution or the set of payoffsn and, thus, achieve the variation that is required for statistical identification while changing the expected value of the lottery equivalently, as required by theory. By design, the mechanism provides the necessary observability in decisions and risk preference. The observability and variation provided by risk preference elicitation makes it a natural

\footnotetext{
${ }^{1}$ In fact, Knight (1921) distinguished risk from uncertainty on the existence of known outcomes and probabilities (see LeRoy and Singell (1987)).

${ }^{2}$ This is consistent with the definition of risk put forth by Rothschild and Stiglitz (1970).
} 
choice to test the hypothesis. ${ }^{3}$

This paper provides the most rigorous test, to date, of the EU prediction that risk averse people prefer changing the probability to changing the reward. The paper presents the results from an experiment in which respondents are presented with two formats of the described risk preference elicitation mechanism, in one of two two decision frames. The formats refer to how the expected values of the lotteries are varied. The expected value of the lotteries in the series varies through either probability variation (PV), where the probability of a reward changes holding the reward constant, or reward variation (RV), where the reward varies holding its probability constant. The decision frames refer to the presentation of the menu of choices. The menu of choices was presented in either ascending order or a random order. Casual comparison of subject choices across frames and formats reveals a preference for increasing the probability relative to increasing the reward in the ascending frame. In the random frame, subjects do not appear to prefer one to the other. This suggests decision frame matters. Structural estimates of the parameter of constant relative risk aversion (CRRA), however, that control for decision error and order effects are statistically equivalent across format and frame. Hence, the apparent framing effect disappears. These estimates indicates that subjects, on average, were risk averse and preferred an increase in the probability to a compensated increase in the reward of a gamble. The results are robust across two of the most common specifications of stochastic error and there is little evidence of probability weighting. Inspection of the data at the individual-level reveals within-format inconsistency is rare but acrossformat inconsistency is substantial. In the ascending order decision frame, $2.83 \%$ and $9.43 \%$ of subjects in the PV and RV formats, respectively, switched from the safe to the risky choice multiple times. Strangely, this type if inconsistency decreased in the

\footnotetext{
${ }^{3}$ Use of this mechanism to test the hypothesis is somewhat controversial. There is some debate regarding the validity of elicited responses. Andersen et al. (2006) find some evidence that the estimates the mechanism elicits are sensitive to the range of lotteries that is presented. Hey and Orme (1994), Ballinger and Wilcox (1997), and Loomes et al. (2002) all find that repeated elicitation on the same respondent yields different risk preferences. These issues are discussed in the conclusion.
} 
random order decision frame; $0.00 \%$ and $7.84 \%$ of subjects in the PV and RV formats, respectively, switched multiple times. ${ }^{4}$ Casual inspection of individual responses across formats indicates roughly $55 \%$ of the sample either over- or under-reacted relative to the theoretical prediction under the assumption of CRRA. ${ }^{5}$

The results have implications beyond the laboratory, most obviously for problems of compliance, such as tax evasion, environmental regulation, corporate governance, and social law. In fact, the theoretical prediction was first discussed by Becker (1968) in his seminal paper on crime and punishment. A regulator has a choice of two instruments to increase compliance: increased monitoring of agents (changing the probability) or increased penalties for non-compliant behavior (changing the outcome). EU theory predicts that increased penalties will have a larger deterrent effect on risk averse agents. ${ }^{6}$ Thus, central to the debate on punishment certainty versus severity is whether risky decisionmaking can be explained reasonably well with EU theory. General findings of directional effects does not directly test EU theory. The experiment presented in this paper directly tests whether both the direction and magnitude of the difference in relative elasticities is consistent with EU theory; formally testing the equivalence of elicited risk preference achieves both. The results from the econometric analysis are consistent with previous findings (Anderson and Stafford, 2003, 2006; Block and Gerety, 1995; Grogger, 1991; Myers, 1983; Witte, 1980). ${ }^{7}$

The paper is organized as follows. Section 2 derives Becker's result and accounts

\footnotetext{
${ }^{4}$ While this multiple switching behavior is referred to as being inconsistent, it may be a signal of indifference, as noted in Andersen et al. (2006). Since an indifference option is not offered, inconsistent preferences cannot be distinguished from indifference.

${ }^{5}$ Assuming the RV CRRA parameter is accurate, $36.94 \%$ (18.47\%) of subjects make too many (few) safe choices in the PV format. Assuming the PV CRRA parameter is accurate, $18.47 \%$ (36.94\%) of subjects make too many (few) safe choices in the RV format.

${ }^{6}$ Assuming an individual is risk averse, then increasing the expected value of a risk by increasing the probability of winning has a greater increase in EU than doing so by increasing the reward. Conversely, decreasing the expected value of a risk by increasing the probability of losing has less of a decrease in EU than doing so by increasing the amount that is lost.

${ }^{7}$ Previous research has focused on losses rather than gains as it relates to punishment certainty versus severity. The general consensus is that people are more sensitive to punishment severity than punishment certainty.
} 
for possible confounding factors. Section 3 describes the experimental design. Section 4 presents the results from the experiment. Section 5 summarizes the results and discusses their implications.

\section{Theoretical Framework}

\subsection{Expected Utility Theory}

Consider a binary lottery that yields a reward $y$ with probability $p$ or 0 with probability $1-p$. The expected value of the lottery is $E V=p y$. Suppose the expected value of the lottery is to be increased, $\Delta E V>0$. This could be accomplished by changing the probability of obtaining the reward, so that $\Delta E V=y \Delta p$, or by changing the size of the reward, so that $\Delta E V=p \Delta y$. Let increasing the expected value by changing the probability be referred to as probability variation (PV) and changing the expected value by changing the reward be referred to as reward variation (RV).

An agent's expected utility $(E U)$ from the lottery is $E U=p U(y)$, where $U(y)$ is a monotonically increasing function of $y .{ }^{8}$ If $U(y)$ is concave, $U^{\prime \prime}(y)<0$, then the agent is risk averse; if $U(y)$ is convex, $U^{\prime \prime}(y)>0$, then the agent is risk seeking; if $U(y)$ is linear, $U^{\prime \prime}(y)=0$, then the agent is risk neutral.

Consider how equivalent changes in the expected value of the lottery affect the EU from the lottery. PV results in a change in EU that is equal to

$$
\Delta E U_{P V}=\Delta p U(y)=\frac{U(y)}{y} \Delta E V
$$

where the second equality uses the result $\Delta p=\frac{\Delta E V}{y}$. RV results in a change in EU that

\footnotetext{
${ }^{8}$ The normalization $U(0)=0$ is assumed throughout the analysis.
} 
is approximately equal to

$$
\Delta E U_{R V}=p U^{\prime}(y) \Delta y=U^{\prime}(y) \Delta E V
$$

where the second equality uses the result $\Delta y=\frac{\Delta E V}{p}$. Thus, for equivalent increases in the expected value of the lottery, the increase in the expected utility from the lottery is greater (lower) with probability variation than with reward variation for risk averse (seeking) agents. ${ }^{9}$

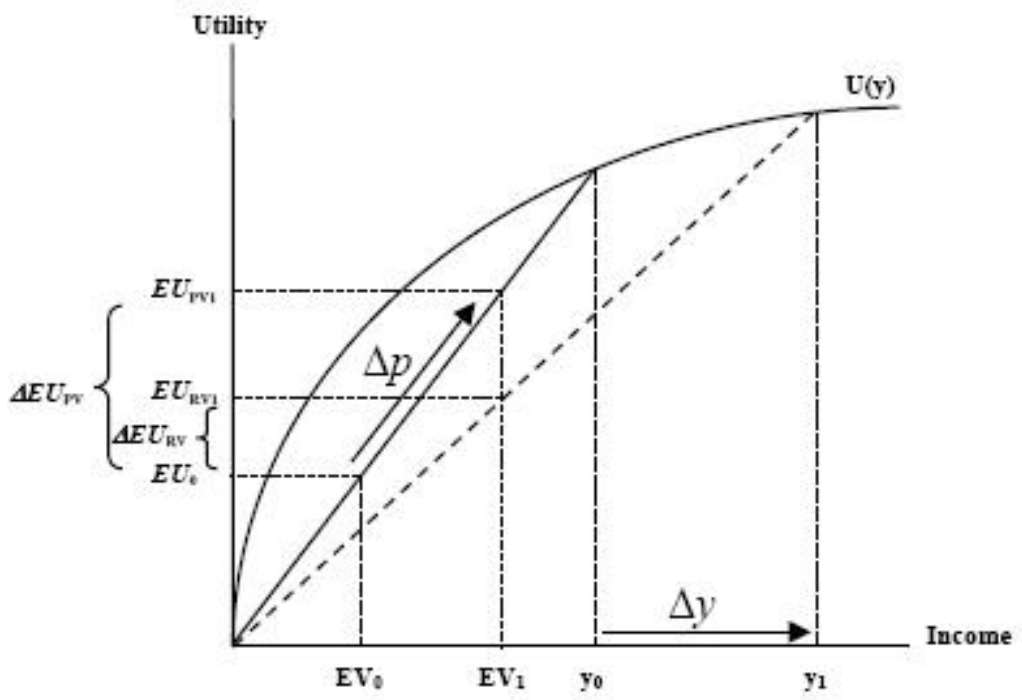

Figure 1: Change in expected utility with PV and RV

The result is shown in Figure 1 for a risk averse agent. Initially, the expected value of the lottery is $E V_{0}=p_{0} y_{0}$, which has a corresponding expected utility of $E U_{0}$. Then the expected value of the lottery is increased by PV, $\Delta p=p_{1}-p_{0}$, and RV, $\Delta y=y_{1}-y_{0}$. The new expected value of the lottery is $E V_{1}=p_{0} y_{1}$ with $\mathrm{RV}$ and $E V_{1}=p_{1} y_{0}$ with PV. The new EU corresponding to the RV lottery is $E U_{R V 1}$ and the new expected utility

\footnotetext{
${ }^{9}$ Becker's (1968) hypothesis pertained to expected sanctions for criminal activity. Thus, his result is the opposite of that shown here, where gains are considered instead of losses.
} 
corresponding to the PV lottery is $E U_{P V 1}$. Since the new EU is greater with $\mathrm{PV}$ than with RV, $E U_{R V 1}<E U_{P V 1}$, the change in the EU is greater with PV than with RV, $\Delta E U_{P V}>\Delta E U_{R V}$

\subsection{Risk Preference Estimation}

The methodology used to elicit risk preferences requires a respondent to make a series, $j=1 \ldots J$, of dichotomous choices. Each decision involves a choice between a binary lottery, where the reward is $y_{j}$ with probability $p_{j}$ and 0 with probability $\left(1-p_{j}\right)$, or a guaranteed amount, $\mu$. The expected value, $E V_{j}=p_{j} y_{j}$, of each lottery $j$ is increased, $E V_{j}>E V_{j-1}$, from $j=2$ to $J$ to induce the respondent choose the lottery over the guaranteed amount for all $j \geq j^{*}$. The decision $j^{*}$ at which the respondent begins choosing the lottery over the guaranteed amount provides an interval estimate of risk preference.

Estimation of risk preference requires specification of a stochastic component to the decision making process. The literature has produced several different approaches to modeling the stochastic error process. To date, the stochastic process has been modeled as a 'trembling hand' (Harless and Camerer, 1994), traditional white noise (Fechner, 1860/1966; Luce, 1959), and random preferences (Becker et al., 1963). By far, the most popular are the Fechner (1860/1966) and Luce (1959) models. ${ }^{10}$ As such, the analysis estimates both models for the comparison between the PV and RV formats.

Assume respondent $i$ 's preferences over potentially random distributions of income

\footnotetext{
${ }^{10}$ The 'trembling hand' approach has rarely been used (Harless and Camerer, 1994; Loomes et al., 2002). The Fechner (1860/1966) approach represents a standard homoskedastic latent variable microeconometric model using 'strong utility'; it is a fairly common approach (Ballinger and Wilcox, 1997; Hey and Orme, 1994; Carbone and Hey, 1994; Hey, 1995; Carbone, 1998; Carbone and Hey, 2000; Loomes et al., 2002; Wilcox, forthcoming). The Luce (1959) white noise model, made popular by Holt and Laury (2002), represents a special case of 'strong utility' known as 'strict utility' and has been used by Dave et al. (2007), Goeree et al. (2003), and Andersen et al. (2008), to name a few. The terminology 'strong utility' and 'strict utility' models stems from decision theory (Debreu, 1958) as noted by Wilcox (2007). Recently, Wilcox (forthcoming) has developed another alternative, 'contextual utility'. Also, random preferences have been used as an alternative to classic microeconometric approaches (Loomes and Sugden, 1995, 1998; Carbone, 1998; Loomes et al., 2002; Wilcox, forthcoming).
} 
are given by the popular constant relative risk aversion (CRRA) utility function where the EU from lottery $j$ is $E U_{i j}=\frac{p_{j} y_{j}^{1-r_{i}}}{1-r_{i}} .{ }^{11}$ Further assume each respondent $i$ maximizes his stochastic EU, $E U_{i j}\left(y_{j k}\right)=p_{j k} U_{i}\left(y_{j k}\right)+\varepsilon_{i k}$, where $\varepsilon_{i k} \sim\left(0, \sigma_{i}\right)$ and $k$ indexes the choices. Let $k=1$ denote the guaranteed amount and $k=0$ denote the lottery. The probability respondent $i$ chooses the guaranteed amount is

$$
\begin{gathered}
\mathrm{P}(\text { Choose } \mu)=P\left[\frac{\mu^{1-r_{i}}}{1-r_{i}}+\varepsilon_{i 1}>\frac{p_{j} y_{j}^{1-r_{i}}}{1-r_{i}}+\varepsilon_{i 0}\right] \\
\mathrm{P}(\text { Choose } \mu)=P\left[\frac{p_{j} y_{j}^{1-r_{i}}-\mu^{1-r_{i}}}{1-r_{i}}<\varepsilon_{i}\right]
\end{gathered}
$$

where $\varepsilon_{i}=\varepsilon_{i 1}-\varepsilon_{i 0}$ is a noise parameter to be estimated. This is the Fechner $(1860 / 1966)$ model of stochastic choice under risk. Following standard probit models, the latent Fechner index, $\frac{E U_{i j}(\mu)-E U_{i j}\left(y_{j}\right)}{\varepsilon_{i}}$, is assumed to be the argument of the cumulative probability density function for the standard normal distribution. Alternatively, the Luce (1959) model, assumes the probability respondent $i$ chooses the guaranteed amount is

$$
\mathrm{P}(\text { Choose } \quad \mu)=\frac{\mu^{\frac{1-r_{i}}{\varepsilon}}}{\mu^{\frac{1-r_{i}}{\varepsilon}}+\left(p_{j} y_{j}^{1-r_{i}}\right)^{\frac{1}{\varepsilon}}}
$$

where $\varepsilon$ is a noise parameter to be estimated. Thus, respondent $i$ 's decision depends on the ratio of the choices rather than the difference between the choices. ${ }^{12}$ Notice the latent Luce index is already defined in terms of a cumulative probability density function. The analysis estimates the models in equations (3) and (4) for both the PV and RV formats

\footnotetext{
${ }^{11}$ The parameter $r_{i}$ measures the risk preference of the respondent, where $r_{i}=0$ if the respondent is risk-neutral; $0<r_{i}$ if the respondent is risk averse; $r_{i}<0$ if the respondent is risk seeking. The choice of a CRRA utility function is based on its popularity and its ability to explain behavior, "under one specific payoff scale, constant relative risk aversion can provide an excellent fit for the data patterns" (Holt and Laury, 2002, pg. 1652). As Ballinger and Wilcox (1997), Hey and Orme (1994), Hey (2001), Hey (2005), and Wilcox (forthcoming) have stressed there is more to be gained from correctly specifying the stochastic process than by introducing additional parameters or new specifications of the structural model. As such, I maintain the CRRA specification under two different specifications of the stochastic process.

${ }^{12}$ Wilcox (2007) discusses the connection between the Fechner (1860/1966) and Luce (1959) models thoroughly.
} 
and then test the equivalence of the risk aversion parameter across formats. Estimation of both models avoids making inferences based on what Wilcox (2007) refers to as a 'stochastic identifying restriction'. ${ }^{13}$

\subsection{Probability Weighting}

The predicted difference in responses between the PV and RV formats is due to the linearity of EU in probability space and non-linearity of EU in income space. There is evidence, however, that suggests preferences are not linear in probabilities (Kahneman and Tversky, 1979; Tversky and Kahneman, 1992; Camerer and Ho, 1994; Prelec, 1998; Gonzalez and Wu, 1999; Stott, 2006). Therefore, we allow for more flexibility in preferences by incorporating a probability weighting function. We assume that $R D E U_{i j}=\pi\left(p_{j}\right) U\left(y_{j}\right)$. That is, we estimate a rank-dependent expected utility function to compliment estimates of expected utility. Following Tversky and Kahneman (1992) we assume the following weighting function:

$$
\pi\left(p_{j}\right)=\frac{p_{j}^{\gamma}}{\left(p_{j}^{\gamma}+\left(1-p_{j}\right)^{\gamma}\right)^{\frac{1}{\gamma}}}
$$

where $p_{j}$ is the probability of getting the reward for lottery $j$ and $\gamma$ represents the curvature parameter. Hence, for $0<\gamma<1(\gamma>1)$ respondents overweight (underweight) small probabilities and underweight (overweight) large probabilities. The previous EU models essentially impose the constraint $\gamma=1$. Estimation of a probability weighting function will permit investigation into the influence of the EU restriction on the comparison between the PV and RV formats. ${ }^{14}$

\footnotetext{
${ }^{13}$ Both Wilcox (2007), Harrison (2007), and Harrison and Rutström (2008) demonstrate that the main finding of Holt and Laury (2002), increasing relative risk aversion, is contingent on their choice of the Luce (1959) model with CRRA since the choice probability is invariant to the scale of payoffs.

${ }^{14}$ Estimation of the probability weighting function is due to a helpful suggestion from an anonymous referee.
} 


\subsection{Decision Frame}

It has been suggested that the menu of choices in the elicitation mechanism may be subject to framing effects (Andersen et al., 2006). That is, when the menu of choices are presented in an ascending order (the most common decision frame), a psychological 'bias towards the middle' may induce a risk averse (seeking) respondent to switch from the safe to the risky choice at a lower (higher) expected value than they may otherwise. It is important to ensure that the experimental results are not confounded by such an effect.

There are two possible remedies: (i) a random order decision frame such that the expected value of the lottery is in random order from one row to the next or (ii) a skewed decision frame that omits lotteries from the menu of decisions (so respondents make fewer decisions and less information regarding preferences is revealed). Andersen et al. (2006) argue that the latter is superior for reasons of cognitive difficulty and noisiness in the data. While there may be validity to their argument, a skewed decision frame changes the bounds on the implied risk preference parameter. Thus, employing a skewed decision frame could result in a difference in elicited risk preference across formats that is strictly due to the change in the implied bounds on the risk preference parameter. Therefore the experiment implements a single random order decision frame in addition to the traditional ascending order decision frame. Assuming risk averse respondents, any psychological 'bias towards the middle' should manifest itself in lower estimates of risk aversion in the ascending menu, which is potentially confounded by such a framing effect, relative to the random menu, which removes the confound.

\section{Experimental Design}

The experiment was conducted to test whether a risk averse respondent is more sensitive to probability variation versus reward variation. EU theory suggests subjects' responses should systematically vary with the elicitation format (i.e. whether the re- 
searcher employs a PV or a RV format). A within-subjects design was used to test the prediction; the same subjects are presented with both formats. In addition, a betweensubjects design was used to control for possible framing effects; subjects are exposed to one of two decision frames. The ascending frame presented lotteries in ascending order while the random frame presented lotteries in a single randomized order.

Table 1: Decisions for PV and RV Formats and Ascending and Random Frames

\begin{tabular}{lcccc}
\hline \hline & \multicolumn{2}{c}{ Ascending Frame } & \multicolumn{2}{c}{ Random Frame } \\
\hline Row & PV Format & RV Format & PV Format & RV Format \\
\hline 1 & $10 \%$ chance of $\$ 10$ & $50 \%$ chance of $\$ 2$ & $70 \%$ chance of $\$ 10$ & $50 \%$ chance of $\$ 8$ \\
2 & $20 \%$ chance of $\$ 10$ & $50 \%$ chance of $\$ 4$ & $30 \%$ chance of $\$ 10$ & $50 \%$ chance of $\$ 10$ \\
3 & $30 \%$ chance of $\$ 10$ & $50 \%$ chance of $\$ 6$ & $40 \%$ chance of $\$ 10$ & $50 \%$ chance of $\$ 16$ \\
4 & $40 \%$ chance of $\$ 10$ & $50 \%$ chance of $\$ 8$ & $90 \%$ chance of $\$ 10$ & $50 \%$ chance of $\$ 4$ \\
5 & $50 \%$ chance of $\$ 10$ & $50 \%$ chance of $\$ 10$ & $100 \%$ chance of $\$ 10$ & $50 \%$ chance of $\$ 14$ \\
6 & $60 \%$ chance of $\$ 10$ & $50 \%$ chance of $\$ 12$ & $20 \%$ chance of $\$ 10$ & $50 \%$ chance of $\$ 6$ \\
7 & $70 \%$ chance of $\$ 10$ & $50 \%$ chance of $\$ 14$ & $10 \%$ chance of $\$ 10$ & $50 \%$ chance of $\$ 12$ \\
8 & $80 \%$ chance of $\$ 10$ & $50 \%$ chance of $\$ 16$ & $50 \%$ chance of $\$ 10$ & $50 \%$ chance of $\$ 18$ \\
9 & $90 \%$ chance of $\$ 10$ & $50 \%$ chance of $\$ 18$ & $80 \%$ chance of $\$ 10$ & $50 \%$ chance of $\$ 20$ \\
10 & $100 \%$ chance of $\$ 10$ & $50 \%$ chance of $\$ 20$ & $60 \%$ chance of $\$ 10$ & $50 \%$ chance of $\$ 2$ \\
\hline \hline
\end{tabular}

Both the PV and RV formats presented subjects with 10 decisions, each required them to choose between a lottery and a guaranteed $\$ 5$. The difference between the formats was the means by which the expected payout of the lottery was changed. Table 1 presents each of the 10 lotteries for the PV and the RV formats under both the ascending order and the random order decision frames. ${ }^{15}$ In the PV format, the reward was held constant at $\$ 10$ while the probability of a reward was varied from $10 \%$ to $100 \%$ in increments of 10\%. In the RV format, the probability of a reward was held constant at $50 \%$ while the reward varied from $\$ 2$ to $\$ 20$ in $\$ 2$ increments. The low reward was held constant at zero in both formats. This was done to make both the expected value and the change in the expected value of the lotteries in the PV and RV formats equivalent in order to be

\footnotetext{
${ }^{15}$ Notice that in the random order decision frame the PV and RV lotteries in a row have different expected values while in the ascending order decision frame the expected values are equal in each row. Hence there are two differences between frames: (i) the order of choices and (ii) the matching of expected values.
} 
consistent with the theoretical argument in the previous section.

In either format, a risk averse subject should switch from choosing the guaranteed $\$ 5$ to choosing the lottery when the expected value of the lottery is greater than $\$ 5$. According to EU theory, the increase in EU from the PV lottery is greater than the increase in EU from the corresponding RV lottery for each expected value from $\$ 6$ to $\$ 10$ for a risk averse subject. This implies a sufficiently risk averse subject should switch to the lottery at a lower expected value in the PV format than in the RV format. ${ }^{16}$

In addition to the the PV and the RV formats, subjects in the ascending order decision frames were required to make decisions in a third format. This format will be referred to as lottery variation (LV). The LV format required subjects to choose between the lotteries in the PV and the RV formats. The purpose of the LV format was not relevant to testing the hypothesis in this paper and therefore the data is not included in the analysis. ${ }^{17}$ It is necessary, however, to acknowledge the potential that a difference in the observed choice pattern between the PV and RV formats could be affected by exposure to the LV format. The experiment was designed to control for this effect.

Experimental sessions consisted of three stages in the ascending order decision frame and two stages in the random order decision frame. In each stage, a different format was presented. As a result each subject was exposed to both the PV and RV formats and made 10 decisions in each. The order in which the formats were presented was randomly assigned to subjects within a session to achieve orthogonal orderings. This randomization controls for possible session effects, such as time of day, as well as any

\footnotetext{
${ }^{16}$ If a subject is not sufficiently risk averse, it may be the case that the difference in EU is insufficient to induce switching sooner in the PV format relative to the RV format. Thus, a risk averse subject may switch to a lottery at the same expected value in both formats given the discrete nature of the elicitation mechanism.

${ }^{17}$ The purpose of the LV format was to test whether risk averse subjects satisfy second-order stochastic dominance. In order to test this hypothesis, it was necessary to verify that subjects were indeed risk averse using subjects' responses in the PV and the RV formats. The EU prediction is that any risk averse individual should choose the RV lottery for decisions 1-4, decision 5 is irrelevant as the choices are identical, and should choose the PV lottery for decisions 6-10. Hence, there is only one pattern of choices in the LV format that is consistent with EU theory, for all risk averse subjects. Thus, this task does not get at the issue of the relative elasticities; it does not generate data that can be used to estimate risk preferences.
} 
Table 2: Experimental Design

\begin{tabular}{|c|c|c|c|c|}
\hline Treatment & \multicolumn{3}{|c|}{ Ascending Decision Frames } & Number of Subjects \\
\hline 1 & Stage $1=\mathrm{PV}$ & Stage $2=\mathrm{LV}$ & Stage $3=\mathrm{RV}$ & 23 \\
\hline 2 & Stage $1=\mathrm{RV}$ & Stage $2=\mathrm{LV}$ & Stage $3=\mathrm{PV}$ & 22 \\
\hline 3 & Stage $1=\mathrm{PV}$ & Stage $2=R V$ & Stage $3=\mathrm{LV}$ & 14 \\
\hline 4 & Stage $1=R V$ & Stage $2=\mathrm{PV}$ & Stage $3=\mathrm{LV}$ & 17 \\
\hline 5 & Stage $1=\mathrm{LV}$ & Stage $2=\mathrm{PV}$ & Stage $3=\mathrm{RV}$ & 15 \\
\hline 6 & Stage $1=\mathrm{LV}$ & Stage $2=R V$ & Stage $3=\mathrm{PV}$ & 15 \\
\hline Treatment & \multicolumn{3}{|c|}{ Random Decision Frames } & Number of Subjects \\
\hline 7 & Stage $1=\mathrm{PV}$ & Stage $2=\mathrm{RV}$ & & 23 \\
\hline 8 & Stage $1=\mathrm{RV}$ & Stage $2=\mathrm{PV}$ & & 28 \\
\hline
\end{tabular}

potential confounding effect that previous formats (including the LV format) may have on the decisions in subsequent formats. For example, if the three formats were presented in the order LV in stage $1, \mathrm{RV}$ in stage 2 , and $\mathrm{PV}$ in stage 3 , the comparison between the $\mathrm{PV}$ and the RV formats could potentially be biased if decisions in stages 2 and 3 were influenced by the exposure to the previous format(s). ${ }^{18}$ Table 2 presents the experimental design.

Prior to making any decisions, subjects were presented with instructions on the computer screen. Subjects were informed in advance that they would be making 10 decisions in each stage. Subjects were told each decision would be between a lottery and another choice, where the computer would use the specified probabilities to determine the outcome of the lottery. Furthermore, subjects were told before they saw any instructions on their screens that only one of their decisions would determine their earnings in the experiment. ${ }^{19}$ The selection of the each subject's decision that determined their payoff was presented as a compound lottery; the computer first selected the stage of the experiment (each had a $\frac{1}{3}$ chance of being selected) and then the decision of the selected stage was

\footnotetext{
${ }^{18}$ The previous evidence of order effects (Harrison et al., 2005; Holt and Laury, 2005) pertains to varying the magnitude of payoffs, which is constant in the experiment.

${ }^{19}$ Specifically, the following script was read aloud to subjects before beginning the instructions. "Before we begin with the instructions, I would like to bring one thing to your attention. As you will read in the instructions, you are going to make several decisions in this experiment. However, only ONE of these will actually determine your earnings for this experiment! So, it is important that you take each decision seriously since a single mistake can be quite costly!"
} 
chosen (each had a $\frac{1}{10}$ chance of being selected). Thus, it is assumed that preferences conform to the Independence Axiom (Samuelson, 1952). The evidence in the literature suggests that 'random lottery selection' is incentive-compatible for simple choice sets (Ballinger and Wilcox, 1997; Starmer and Sugden, 1991; Wilcox, 1993). ${ }^{20}$

Subjects were given instructions pertaining to the stage (which reiterated much of the general instructions) and shown an example decision screen prior to making any decisions for a particular stage. ${ }^{21}$ Upon completion of the decisions in a stage, subjects moved on to the subsequent stage. After completion of the final stage, subjects were shown the stage and the decision that was selected by the computer for payment, as well as the outcome of the lottery if chosen. Subjects were paid individually in private after completing some demographic and debriefing questions. ${ }^{22}$ At no time was any deception used in the experiment.

The experiment was conducted at the University of Calgary. The subject pool is composed of volunteer students at the university. Subject's were recruited by email via the lab's Online Recruitment System for Experimental Economics (ORSEE) (Greiner, 2004). The experiment was programmed and conducted with the software Z-Tree (Fischbacher, 2007). Experimental sessions lasted approximately 35 minutes and average earnings were $\$ 12$ including a $\$ 5$ show-up fee. A total of 157 subjects participated; 106 subjects participated in the ascending order decision frame and 51 subjects participated in the random order decision frame.

\footnotetext{
${ }^{20}$ See Harrison and Rutström (2008) for a discussion of the costs and benefits associated with the 'random lottery selection' procedure.

${ }^{21}$ The example decision screen displayed the exact decisions the subject would have to make on the subsequent screen.

${ }^{22}$ Subjects were asked questions to verify whether the necessary prerequisites to induce values were satisfied (Friedman and Sunder, 1994, p. 13). Responses were indicated on likert scales. Nearly $80 \%$ of respondents indicated the highest level of understanding (to verify salience). $52 \%$ of subjects indicated that maximizing their own gains was of the highest level of importanc (to verify monotonicity). $54 \%$ of subjects indicated that minimizing the experimenter's losses was of the lowest level of importance (to verify dominance).
} 


\section{Analysis and Results}

EU theory implies sufficiently risk averse subjects should make fewer safe choices in the PV format relative to the RV format. The analysis begins by comparing the proportion of subjects that chose the guaranteed $\$ 5$, for each decision across formats and frames. Then the data is used to construct bounds on the implied CRRA parameter for each subject to determine the distribution of risk preferences. These interval estimates are used to investigate, at the individual level, the degree of consistency with EU theory. Finally, using the microeconometric framework described in Section 2.2, a test for a difference in the average CRRA parameter across the formats and decision frames is conducted. A statistical equivalence in the estimated CRRA parameter across formats suggests subjects are being consistent with the behavior implied by 'noisy' EU maximization.

\subsection{Comparison of Safe Choices in PV and RV formats}

Figure 2 plots the proportion of the sample that chose the safe choice for each decision in both the PV and the RV formats. The left panel presents the data for the ascending order decision frame and the right panel presents the data from the random order decision frame. For the ascending order decision frame, a lower proportion of subjects chose the guaranteed $\$ 5$ over the lottery in the PV format relative to the RV format in the region where risk averse subjects should switch (expected values 6 - 10). ${ }^{23}$ This pattern of choices is consistent with the prediction that the EU from the lottery is increasing more under PV relative to RV for a risk averse subject. The result disappears, however, when the random order decision frame is plotted, as can be seen in the right panel. The proportion of subjects that chose the guaranteed $\$ 5$ for each decision is virtually identical across formats. This pattern of choices is indicative of some sort of framing effect.

Two-sample Wilcoxon signed-rank tests are conducted on the distribution of choices for each decision. This is a nonparametric test of the hypothesis that the distributions

\footnotetext{
${ }^{23}$ The decision number is equivalent to the expected value of the lottery in both formats.
} 

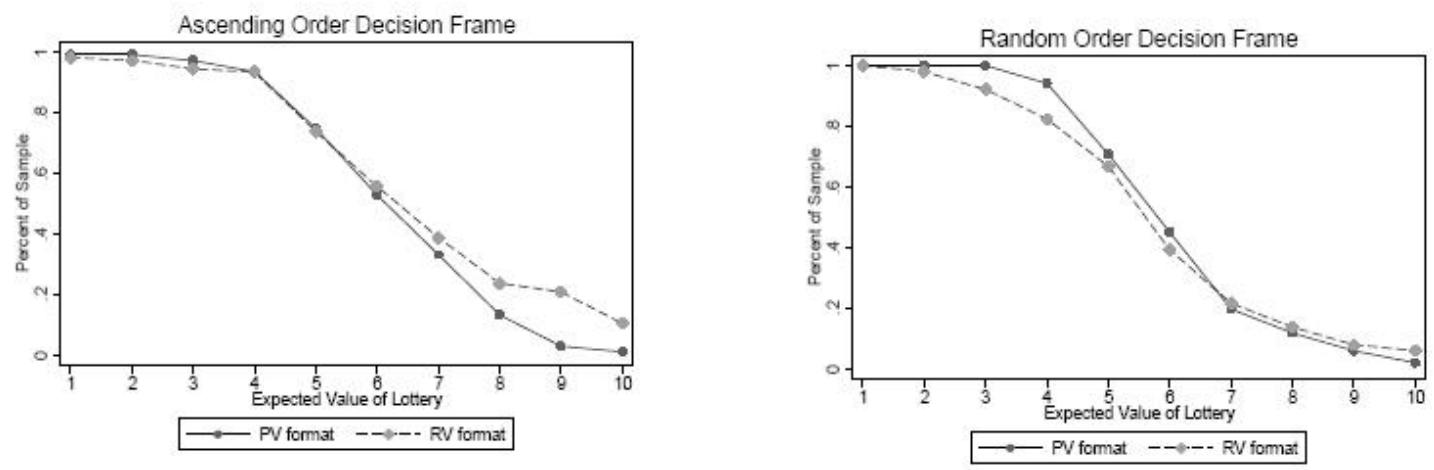

Figure 2: Proportion of sample choosing the guaranteed amount.

of matched pairs of observations are the same. ${ }^{24}$ The test statistics for equivalence in the proportion of safe choices between the two formats in the ascending order decision frame for expected values $\$ 8, \$ 9$, and $\$ 10$ all reject the null hypothesis at a $5 \%$ level of significance. $^{25}$ Thus, the distributions of choices in the two formats for the ascending order decision frame are statistically different from each other in the direction that is consistent with the theoretical prediction. This result disappears when the random order decision frame is analyzed, as the test statistics are no longer significant. ${ }^{26}$

Table 3: Risk Preference Classification Based on Lottery Choices

\begin{tabular}{ccccccc}
\hline \hline $\begin{array}{c}\text { Number of } \\
\text { Safe Choices }\end{array}$ & $\begin{array}{c}\text { PV Risk } \\
\text { Parameter Range }\end{array}$ & $\begin{array}{c}\text { RV Risk } \\
\text { Parameter Range }\end{array}$ & $\begin{array}{c}\text { PV } \\
\text { Ascend }\end{array}$ & $\begin{array}{c}\text { PV Random } \\
\text { Rand Sample }\end{array}$ & $\begin{array}{c}\text { RV } \\
\text { Ascend }\end{array}$ & $\begin{array}{c}\text { RV } \\
\text { Random }\end{array}$ \\
\hline or less & $-\infty \quad<\mathrm{r} \leq-0.737$ & $-\infty \quad<\mathrm{r} \leq-2.802$ & 1.89 & 0.00 & 2.83 & 5.88 \\
3 & $-0.737<\mathrm{r} \leq-0.322$ & $-2.802<\mathrm{r} \leq-0.475$ & 2.83 & 5.88 & 1.89 & 9.80 \\
4 & $-0.322<\mathrm{r} \leq 0.000$ & $-0.475<\mathrm{r} \leq 0.000$ & 18.87 & 23.53 & 16.98 & 19.61 \\
5 & $0.000<\mathrm{r} \leq 0.263$ & $0.000<\mathrm{r} \leq 0.208$ & 26.42 & 25.49 & 22.64 & 25.49 \\
6 & $0.263<\mathrm{r} \leq 0.485$ & $0.208<\mathrm{r} \leq 0.327$ & 17.92 & 25.49 & 19.81 & 21.57 \\
7 & $0.485<\mathrm{r} \leq 0.678$ & $0.327<\mathrm{r} \leq 0.404$ & 19.81 & 7.84 & 13.21 & 3.92 \\
8 or more & $0.678<\mathrm{r} \leq 1.000$ & $0.404<\mathrm{r} \leq 1.000$ & 12.27 & 11.76 & 22.63 & 13.72 \\
\hline \hline
\end{tabular}

\footnotetext{
${ }^{24}$ This test accounts for the fact that the observations in both formats are based on the choices of the same subjects. Thus, the two samples are not independent.

${ }^{25}$ The test statistics are are $z=-2.40, z=-4.36$, and $z=-3.16$, respectively. The test statistics for decisions 9 and 10 actually reject the null hypothesis at the $1 \%$ level of significance. If the data is pooled across treatments 3 and 4 (to minimize any influence of the LV format) the test statistics for decisions $7,8,9$, and 10 are $z=-2.121, z=-2.121, z=-3.606$, and $z=-2.646$, respectively. These all reject the null hypothesis at the $5 \%$ level of significance.

${ }^{26}$ The only statistically significant difference between the formats in the random order decision frame occurs at expected values of $\$ 3$ and $\$ 4$ where the test statistics are $z=2.000$ and $z=2.449$, respectively.
} 
Since subjects make 10 decisions in each format, bounds on the implied risk aversion parameter can be constructed based on the number of safe choices. Table 3 shows the ranges of the implied risk aversion parameter in columns 2 and 3 for the PV and the RV formats, respectively, assuming a CRRA utility function. The table indicates that the majority of subjects were risk averse. Thus, risk aversion is a likely explanation for the difference in choices displayed in the left panel of figure 2, as EU theory suggests. While the distribution of risk preferences clearly changes across decision frames, the change is not consistent with a psychological 'bias towards the middle'. In either the PV or the RV format, the distribution of risk preferences is more concentrated towards the middle (4, 5 , or 6 safe choices) in the random order decision frame than the ascending order decision frame.

Table 4: Number of Safe Choices in PV and RV Formats

\begin{tabular}{ccccccccccccc}
\hline \hline Number of Safe & \multicolumn{1}{c}{ Number of Safe Choices in RV } \\
Choices in PV & 0 & 1 & 2 & 3 & 4 & 5 & 6 & 7 & 8 & 9 & 10 & Total \\
\hline 0 & 0 & 0 & 0 & 0 & 0 & 0 & 0 & 0 & 0 & 0 & 0 & 0 \\
1 & 0 & 0 & 0 & 0 & 0 & 0 & 0 & 0 & 0 & 0 & 0 & 0 \\
2 & 0 & 0 & $\mathbf{1}$ & 0 & 0 & $\mathbf{1}$ & 0 & 0 & 0 & 0 & 0 & 2 \\
3 & 0 & 0 & $\mathbf{2}$ & 2 & 1 & $\mathbf{1}$ & 0 & 0 & 0 & 0 & 0 & 6 \\
4 & $\mathbf{1}$ & 0 & $\mathbf{1}$ & $\mathbf{3}$ & 15 & $\mathbf{7}$ & $\mathbf{3}$ & $\mathbf{1}$ & $\mathbf{1}$ & 0 & 0 & 32 \\
5 & 0 & 0 & $\mathbf{1}$ & $\mathbf{2}$ & $\mathbf{6}$ & 18 & $\mathbf{1 0}$ & $\mathbf{1}$ & $\mathbf{1}$ & $\mathbf{1}$ & $\mathbf{1}$ & 41 \\
6 & 0 & 0 & 0 & 0 & $\mathbf{2}$ & $\mathbf{8}$ & 14 & 3 & 3 & 1 & $\mathbf{1}$ & 32 \\
7 & 0 & 0 & 0 & 0 & $\mathbf{3}$ & $\mathbf{2}$ & $\mathbf{3}$ & $\mathbf{7}$ & $\mathbf{4}$ & 3 & 3 & 25 \\
8 & 0 & 0 & 0 & 0 & $\mathbf{1}$ & 0 & $\mathbf{2}$ & $\mathbf{4}$ & $\mathbf{1}$ & $\mathbf{4}$ & 2 & 14 \\
9 & 0 & 0 & 0 & 0 & 0 & 0 & 0 & 0 & 0 & $\mathbf{2}$ & 2 & 4 \\
10 & 0 & 0 & 0 & 0 & 0 & 0 & 0 & 0 & 0 & 0 & $\mathbf{1}$ & 1 \\
\hline Total & 1 & 0 & 5 & 7 & 28 & 37 & 32 & 16 & 10 & 11 & 11 & 157 \\
\hline \hline
\end{tabular}

It is worthwhile to investigate the extent to which the aggregate results are representative of individual subject behavior. Table 4 summarizes the the number of safe choices made by each subject in the PV and the RV formats. Using the ranges of the implied CRRA parameter from table 3, individual inconsistencies across formats can be identified, as indicated by bold numbers. These are subjects whose implied CRRA parameters do not overlap across formats. Italic numbers indicate subjects that consistently revealed preferences across the two formats, or at least the implied CRRA parameters overlap. 
Indeed there are a large number of inconsistencies. Approximately $58 \%$ of the sample either over- or under-reacted relative to the theoretical prediction under the assumption of CRRA. ${ }^{27}$ Despite the inconsistencies across formats, there were few inconsistencies within formats. $1.91 \%$ of subjects in the PV format and $8.92 \%$ of subjects in the RV format switched multiple times. ${ }^{28}$ Still, the question remains, can these inconsistencies be explained by noise in the decision process?

\subsection{Estimated CRRA Risk Parameter}

The micoreconometric models presented in Section 2.2 are used to test whether estimates of the average CRRA parameter are statistically equivalent across the two formats. The estimates under both models of the stochastic error process are reported. The estimation results for the Fechner (1860/1966) model from equation (3) are shown in the first and second columns of table 5. The estimation results for the Luce (1959) model from equation (4) are shown in the third and fourth columns of table 5 . The first and third columns report estimates on data pooled across both formats and frames. The second and fourth columns report estimates of the CRRA parameter when it is allowed to vary across formats and frames. Dummy variables to indicate format and frame combinations are included. ${ }^{29}$ The baseline case, captured by the constant term, is the PV format with an ascending order decision frame. All models are estimated using maximum likelihood assuming clustered errors to account for repeated observations on the same subject. ${ }^{30}$

The estimation results in the second and fourth columns demonstrate the effects of

\footnotetext{
${ }^{27}$ Assuming the RV CRRA parameter is accurate, $39.49 \%$ (18.47\%) of subjects make too many (few) safe choices in the PV format. Assuming the PV CRRA parameter is accurate, $18.47 \%$ (39.49\%) of subjects make too many (few) safe choices in the RV format.

${ }^{28} \mathrm{In}$ treatments 3 and 4 (the ascending frame), $3.23 \%$ and $22.58 \%$ of subjects in the PV and RV formats, respectively, switched multiple times. Strangely, this type if inconsistency decreased in treatments 7 and 8 (the random frame); $0.00 \%$ and $7.84 \%$ of subjects in the PV and RV formats, respectively, switched multiple times. Again, this multiple switching behavior is referred to as being inconsistent, although this may be a signal of indifference, as noted in Andersen et al. (2006).

${ }^{29}$ The analysis also investigated the influence of demographics and order effects. Demographics were not significant. Order effects are controlled for in the analysis but are not reported.

${ }^{30} \mathrm{~A}$ detailed discussion of the estimation technique is discussed in Harrison (2007).
} 
Table 5: Maximum Likelihood Estimation of CRRA Utility

\begin{tabular}{|c|c|c|c|c|c|c|}
\hline & \multicolumn{4}{|c|}{ No Probability Weighting } & \multicolumn{2}{|c|}{ Probability Weighting } \\
\hline & Fechner & Fechner & Luce & Luce & Fechner & Luce \\
\hline CRRA & & & & & & \\
\hline Constant & $\begin{array}{c}0.245^{* * *} \\
(0.038)\end{array}$ & $\begin{array}{c}0.291^{* * *} \\
(0.060)\end{array}$ & $\begin{array}{c}0.197^{* * *} \\
(0.047)\end{array}$ & $\begin{array}{c}0.244^{* * *} \\
(0.073)\end{array}$ & $\begin{array}{c}0.232^{* * *} \\
(0.041)\end{array}$ & $\begin{array}{c}0.193^{* * *} \\
(0.046)\end{array}$ \\
\hline RV Ascending & & $\begin{array}{l}-0.020 \\
(0.025)\end{array}$ & & $\begin{array}{c}-0.003 \\
(0.030)\end{array}$ & & \\
\hline PV Random & & $\begin{array}{l}-0.009 \\
(0.090)\end{array}$ & & $\begin{array}{l}-0.026 \\
(0.101)\end{array}$ & & \\
\hline RV Random & & $\begin{array}{l}-0.070 \\
(0.076)\end{array}$ & & $\begin{array}{l}-0.099 \\
(0.096) \\
\end{array}$ & & \\
\hline $\begin{array}{c}\text { Noise } \\
\text { Parameter }\end{array}$ & & & & & & \\
\hline Constant & $\begin{array}{c}0.905^{* * *} \\
(0.032)\end{array}$ & $\begin{array}{c}0.943^{* * *} \\
(0.037) \\
\end{array}$ & $\begin{array}{c}0.167^{* * *} \\
(0.017)\end{array}$ & $\begin{array}{c}0.167^{* * *} \\
(0.017)\end{array}$ & $\begin{array}{c}0.959^{* * *} \\
(0.066)\end{array}$ & $\begin{array}{c}0.164^{* * *} \\
(0.016) \\
\end{array}$ \\
\hline $\begin{array}{l}\text { Weighting } \\
\text { Parameter }\end{array}$ & & & & & & \\
\hline Constant & & & & & $\begin{array}{c}1.202^{* * *} \\
(0.100)\end{array}$ & $\begin{array}{c}0.935^{* * *} \\
(0.076) \\
\end{array}$ \\
\hline Log Likelihood & -1060.671 & -1057.959 & -1055.162 & -1052.262 & -1045.999 & -1054.684 \\
\hline
\end{tabular}

Notes: Standard errors are reported in parenthesis. Significance levels are indicated by asterisks: *** : 1\%. All estimates are based on 3140 observations; 20 decisions for each of the 157 subjects. All models include treatment dummies on the CRRA parameter, to control for order effects, that are not reported. While none are individually significant in models 2 , there are significant order effects in models 1 and with probability weighting. A likelihood ratio indicates that format and frame effects are jointly insignificant.

format and frame on elicited risk preferences. There is no statistical difference in the estimated average CRRA parameter across formats, as indicated by the insignificance of the estimated coefficient on the dummy variables for the RV format with an ascending order decision frame and those for the PV and RV formats in the random order decision frame. This also implies there is no statistical difference in the estimated average CRRA parameter across decision frames, although these are different subjects. ${ }^{31}$ Furthermore, statistical significance aside, the estimated coefficients are all negative. Recall, a psychological 'bias towards the middle' should manifest itself in lower estimates of risk aversion in the ascending frame. The estimated parameters, however, indicate that the random

\footnotetext{
${ }^{31}$ Still both samples come from the same pool of student volunteers. Thus it is reasonable to expect the sample means to be equivalent.
} 
frame elicited a lower average CRRA parameter, as indicated by the negative signs on the dummy variables for the random order decision frame. Hence, the data reject the hypothesis of a psychological 'bias towards the middle'. Thus, after allowing for a stochastic error process and controlling for order effects, the consistency of responses across formats in the ascending order also holds in the random order decision frame. ${ }^{32}$

Finally, the analysis investigates the extent to which the results are influenced by the EU restriction on the probability weighting parameter in equation (5). By design, the PV format has no variation in payoffs and the RV format has no variation in probabilities. The lack of variation makes simultaneous estimation of the curvature of the utility function and the curvature of the probability weighting function impossible. In order to obtain sufficient variation in the parameters of interest, the data is pooled across formats and frames and estimate the structural parameters under both the Fechner $(1860 / 1966)$ and Luce (1959) models. The results are reported in columns 5 and 6 of table 5, respectively. As can be seen, the estimated average CRRA parameters are quite close to the corresponding estimates without probability weighting reported in columns 1 and 3 , respectively. Furthermore, the estimated probability weighting parameters are close to unity. Thus, to the extent that probability weighting occurs, it does not appear to be severe.

\section{Discussion}

The question posed at the outset of this paper asked whether risk averse individuals prefer an increase in the expected value of a lottery due to increasing the probability of winning to doing so by increasing the reward? The answer is a qualified yes. Casual comparison of subject choices in figure 2 reveals a preference for increasing the probability

\footnotetext{
${ }^{32}$ Note that the estimated noise parameter is intended to capture noise in the individual decisionmaking process. Given the extremely low rates of individual within-format inconsistency, there is practically no noisiness in the data. This is reflected in the fact that the estimated Fechner noise parameter is less than one and the estimated Luce noise parameter is close to zero.
} 
of winning in the ascending frame, although subjects in the random frame do not appear to prefer one to the other. Structural estimates of the CRRA parameter, that control for decision error and order effects, however, are statistically equivalent across formats and frames. These estimates indicate subjects were, on average, risk averse and preferred an increase in the probability to an increase in the reward. The results are robust across the two most popular models stochastic error and do not appear to be severely influenced by probability weighting.

The results have implications beyond the laboratory, most notably for compliance problems. Whether increasing the certainty of apprehension or the severity of punishment, if apprehended, is the larger deterrent is still an issue of debate. ${ }^{33}$ To date, empirical studies of naturally occurring individual-level data have been limited to released arrestees (Grogger, 1991; Myers, 1983; Witte, 1980). It is not clear that released arrestees are the appropriate sample to address the question. As Grogger (1991) points out, individuals who have been imprisoned may have such poor labor market opportunities that they will prefer criminal activities regardless of the enforcement regime. As such, the results from these studies have been mixed (Witte, 1980; Myers, 1983; Grogger, 1991).

In an effort to reconcile differences between criminals and the general population, Block and Gerety (1995) conducted a novel experiment that analyzed the behavior of university students relative to convicted felons in a cartel game. They found that felons are more responsive to punishment certainty, in agreement with Witte (1980) and Grogger (1991), while students are more sensitive to punishment severity, as has been cited Anderson and Stafford (2003, 2006). This evidence suggests that criminals have a preference for risk while the general population, as represented by university students, is risk averse. When Block and Gerety (1995) elicited the risk preferences of convicts and

\footnotetext{
${ }^{33}$ As noted by Polinsky and Shavell (2000, p. 73) in their survey of the economic literature on law enforcement, "Empirical work on law enforcement is strongly needed to better measure the deterrent effects of sanctions, especially to separate the influence of the magnitude of sanctions from their probability of application."
} 
students, however, they found no difference between the two groups. ${ }^{34}$ Furthermore, the general finding of directional effects, while important does not directly test EU theory. Individuals may still under- or overreact relative to EU predictions. From table 4, approximately $58 \%$ of subjects in the experiment either over- or under-reacted relative to the theoretical prediction under the assumption of CRRA. Assuming the PV CRRA parameter is accurate, $18.47 \%(39.49 \%)$ of subjects make too many (few) safe choices in the RV format. Conversely, assuming the RV CRRA parameter is accurate, $39.49 \%$ (18.47\%) of subjects make too many (few) safe choices in the PV format. Still, the microeconometric analysis suggests subject behavior, on average, is consistent with previous findings (Anderson and Stafford, 2003, 2006; Block and Gerety, 1995; Grogger, 1991; Myers, 1983; Witte, 1980).

The design employed in this experiment carefully manipulated the decision frame. Andersen et al. (2006) provide some evidence of a framing effect based on a skewed version of the menu of lottery choices. In their review of the literature, Harrison and Rutström (2008, p. 47) state "that there may be some slight framing effect, but it is not systematic ...". The results from this experiment are consistent with this assessment; they reveal some slight framing effect but it does not appear to be systematic either. Clearly, the data do not support a psychological 'bias towards the middle'. In the random frame, intended to remove such a confound, the distribution of risk preferences is actually more concentrated towards the middle. This is confirmed by the negative effect, albeit insignificant, of the random frame on the estimated CRRA parameter.

Finally, the percentage of subjects that switch from safe to risky multiple times within a format is quite low in either decision frame. Hey and Orme (1994), Ballinger and Wilcox (1997), and Loomes et al. (2002) all report a significant amount of multiple switching behavior when they present lottery choices individually and in random order. Andersen

\footnotetext{
${ }^{34}$ Block and Gerety (1995) use hypothetical questions to elicit risk preference which raises the issue of validity given the lack of salience (Smith and Walker, 1993). Holt and Laury (2002) provide evidence that hypothetical choices do not induce subjects to truthfully reveal their preferences.
} 
et al. (2006) observe a significant reduction in multiple switching behavior when they include an indifference option with the presentation of choices as a menu; suggesting such behavior is a signal of indifference. This experiment results in a similarly low rate of multiple switching behavior through the use of additional verbal instruction, emphasizing the random lottery selection procedure, before the experiment began combined with the presentation of choices as a menu. ${ }^{35}$ Hopefully future research will provide a systematic investigation into the nature of multiple switching behavior, as this will likely shed light on the nature of the stochastic error process. Overall, the risk preference elicitation mechanism used in this experiment appears to be robust not only to variation in format, but also variation in frame.

\footnotetext{
${ }^{35}$ Pooling the data across decision frames, $1.91 \%$ in the PV format and $8.92 \%$ of subjects in the RV format switched multiple times.
} 


\section{References}

Andersen, S.G., G. Harrison, M.I. Lau, and E.E. Rutström, "Elicitation Using Multiple Price List Formats," Experimental Economics, 2006, 9 (4), 383 - 406.

_ , _ , , and _ , "Eliciting Risk and Time Preferences," Econometrica, 2008, 76 (583 $-618)$.

Anderson, L.R. and S.L. Stafford, "Punishment in a Regulatory Setting: Experimental Evidence from the VCM," Journal of Regulatory Economics, 2003, 24, 91 110.

_ and _, "Does Crime Pay? A Classroom Demonstration of Monitoring and Enforcement," Southern Economic Journal, 2006, 72, 1016 - 1025.

Ballinger, T.P. and N.T. Wilcox, "Decisions, Error and Heterogeneity," The Economic Journal, July 1997, 10\%, 1090 - 1105.

Becker, G., M. DeGroot, and J. Marschak, "Stochastic Models of Choice Behavior," Behavioral Science, 1963, 8, 41 - 55.

Becker, G.S., "Crime and Punishment: An Economic Approach," Journal of Political Economy, 1968, 76, $169-217$.

Block, M.K. and V.E. Gerety, "Some Experimental Evidence on Difference between Student and Prisoner Reactions to Monetary Penalties and Risk," Journal of Legal Studies, 1995, 24, 123 - 138.

Camerer, C.F. and T-H Ho, "Violations of the Betweeness Axiom and Nonlinearity in Probabilities," Journal of Risk and Uncertainty, 1994, 8, 167 - 196.

Carbone, E., "Investigation of Stochastic Preference Theory Using Experimental Data," Economics Letters, 1998, 57, 305312.

- and J.D. Hey, "Discriminating Between Preference Functionals - A Preliminary Monte Carlo Study," Journal of Risk and Uncertainty, 1994, 8, 223 - 242.

_ and _, "Which Error Story is Best," Journal of Risk and Uncertainty, 2000, 20, 161 -176 .

Dave, C., C. Eckel, C. Johnson, and C. Rojas, "On the Heterogeneity, Stability, and Validity of Risk Preferences," Unpiblished Manuscript, University of Texas at Dallas, 2007.

Debreu, G., "Stochstic Choice and Cardinal Utility," Econometrica, 1958, 26, 440 444.

Eckel, C.C. and R.R. Wilson, "Is Trust a Risky Decision," Journal of Economic Behavior and Organiziation, 2004, 55, $447-465$. 
Fechner, G., Elements of Psychophysics, New York: Holt, Rinehart and Winston, 1860/1966.

Fischbacher, U., "Z-Tree - Zurich Toolbox for Readymade Economic Experiments Experimenter's Manual," Experimental Economics, 2007, 171 - 178.

Friedman, D. and S. Sunder, Experimental Methods: A Primer for Economists, New York, NY: Cambridge University Press, 1994.

Goeree, J., C.A. Holt, and T.R. Palfrey, "Risk Averse Behavior in Generalized Matching Pennies Games," Games and Economic Behavior, 2003, 45, 97 - 113.

Gonzalez, R. and G. Wu, "On the Shape of the Probability Weighting Function," Cognitive Psycology, 1999, 38, 129 - 166.

Greiner, B., "The Online Recruitment System ORSEE 2.0 - A Guide for the Organization of Experiments in Economics," Working Paper Series in Economics 10, 2004, University of Cologne.

Grogger, Jeffrey, "Certainty VS. Severity of Punishment," Economic Inquiry, 1991, 29, $297-309$.

Harless, D.W. and C.F. Camerer, "The Predictive Utility of Generalized Expected Utility Theories," Econometrica, 1994, 62 (6), 1251 - 1289.

Harrison, G.W., "Maximum Likelihood Estimation of Utility Functions using Stata," Working Paper 06-12, Department of Economics, College of Business Administration, University of Central Florida, 2007.

- and E.E. Rutström, Research in Experimental Economics, Vol. 12, Emerald Group Publishing Limited,

_ , E. Johnson, M.M. McInnes, and E.E. Rutström, "Risk Aversion and Incentive Effects: Comment," American Economic Review, 2005, 95 (3), 897 - 901.

Hey, J.D., "Experimental Investigations of Errors in Decision-Making Under Uncertainty," European Economic Review, 1995, 29, 633 - 640.

_ , "Does Repetition Improve Consistency?," Experimental Economics, 2001, 4, 5- 54.

_ , "Why We Should Not Be Silent About Noise," Experimental Economics, 2005, 8, 325 -345 .

- and C. Orme, "Investigating Generalizations of Expected Utility Theory Using Experimental Data," Econometrica, November 1994, 62 (6), 1291 - 1326.

Holt, C.A. and S.K. Laury, "Risk Aversion and Incentive Effects," American Economic Review, 2002, 92 (5), 1644 - 1657. 
_ and _, "Risk Aversion and Incentive Effects New Data without Order Effects," American Economic Review, 2005, 95 (3), 902 - 904.

Kahneman, D. and A. Tversky, "Prospect Theory An Analysis of Decision Under Risk," Econometrica, 1979, 47, 263 - 291.

Knight, F.H., Risk, Uncertainty and Profit, Boston: Houghton Mifflin, 1921.

LeRoy, S.F. and L.D. Singell, "Knight on Risk and Uncertainty," Journal of Political Economy, 1987, 95 (2), $394-406$.

Loomes, G. and R. Sugden, "Incorporating a Stochastic Element Into Decision Theories," European Economic Review, 1995, 39, 641 - 648.

_ and _ , "Testing Alternative Stochastic Specifications for Risky Choice," Economica, $1998,65,581-598$.

_ , P.G. Moffatt, and R. Sugden, "A Microeconometric Test of Alternative Stochastic Theories of Risky Choice," Journal of Risk and Uncertainty, 2002, 24, 103 - 130.

Luce, D., Individual Choice Behavior, New York: John Wiley \& Sons, 1959.

Myers, S.B, "Estimating the Economic Model of Crime: Employment versus Punishment Effects," Quarterly Journal of Economics, February 1983, pp. 157 - 166.

Polinsky, A.M. and S. Shavell, "The Economic Theory of Public Enforcement of Law," Journal of Economic Literature, 2000, 38, 45 - 76.

Prelec, D., "The Probability Weighting Function," Econometrica, 1998, 66, 497 - 528.

Rothschild, M. and J. Stiglitz, "Increasing Risk: I. A Definition," Journal of Economic Theory, 1970, 2, 225 - 243.

Samuelson, P.A., "Probability, Utility, and the Indedendence Axiom," Econometrica, 1952, 20 (4), $670-678$.

Smith, V.L. and J. Walker, "Monetary Rewards and Decision Cost in Experimental Economics," Economic Inquiry, 1993, pp. 245 - 261.

Starmer, C. and R. Sugden, "Does the Random-Lottery Incentive System Elicit True Preferences? An Experimental Investigation," American Economic Review, September 1991, 81 (4), $971-978$.

Stott, H.P., "Cumulative Prospect Theory's Functional Managerie," Journal of Risk and Uncertainty, 2006, 32, $101-130$.

Tversky, A. and D. Kahneman, "Advances in Prospect Theory: Cumulative Representation of Uncertainty," Journal of Risk and Uncertainty, 1992, 5, 297 - 323.

Wilcox, N.T., "Lottery Choice: Incentives, Complexity, and Decision Time," The Economic Journal, 1993, 103, 1397 - 1470. 
_ , Risk Aversion in Experiments, Vol. 12 of Research in Experimental Economics, Greenwich, CT: JAI Press, 2007.

_, "'Stochastically More Risk Averse:' A Contextual Utility Approach," Journal of Econometrics, forthcoming.

Witte, A.D., "Estimating the Economic Model of Crime with Individual Data," Quarterly Journal of Economics, February 1980, pp. 57 - 84. 